\title{
Arquitectura de un Laboratorio Remoto Desde el Enfoque DE LA FORMACIÓN DE INGENIEROS EN EAD
}

\author{
Architecture of a Remote from a engineer training In DE \\ APPROACH
}

\author{
Pérez Waltero Harold Esneider \\ Universidad Nacional Abierta y a Distancia -UNAD, Escuela de Ciencias Básicas, \\ Tecnología e Ingeniería -ECBTI, Bogotá, D.C., Colombia \\ harold.perez@unad.edu.co
}

\section{RESUMEN}

La Educación Abierta y a Distancia, EaD, soportada en las Tecnologías de la Información y la Comunicación TIC, se proyecta como una de las mejores alternativas para responder de manera pertinente al nuevo contexto de globalización, en procura de generar una sociedad incluyente, desarrollada y competitiva. Sin embargo, es necesario avanzar en el desarrollo de recursos tecnológicos que permitan garantizar procesos formativos de calidad a nivel de Ingeniería bajo esta modalidad, dado que aún persisten cuestionamientos al respecto. Son varios los aspectos que se pueden abordar para evidenciar la calidad con la que se puede desarrollar un proceso de formación soportado en las Tecnología de la Información y la Comunicación, TIC. Uno de los aspectos estructurales en el proceso formativo de ingenieros en esta metodología, es la práctica de laboratorio; desde esta perspectiva se plantea un escenario real de práctica, aunque remoto, denominado Laboratorio de Acceso Remoto o Laboratorio Remoto (LR). En este sentido, lo que se plantea es el diseño de una arquitectura a nivel de ingeniería para LR, que elimine la barrera de espacio y tiempo, reduzca costos en infraestructura y tenga un mayor grado de universalidad en el acceso, sin sacrificar la calidad que demanda el ejercicio riguroso de la práctica de laboratorios en la formación del Ingeniero.

Palabras clave: arquitectura, competitividad, educación a distancia, ingeniería, globalización, protocolo, TIC.

\section{Abstract}

The Open and Distance Education, EaD, supported by Information and Communication Technologies ICT, is projected as one of the best alternatives to respond in ways that are relevant to the new context of globalization, seeking to generate an inclusive, developed and competitive society. Nevertheless, it is necessary to advance in the development of technological 
resources that allow to guarantee formative quality processes at Engineering level under this form, since questions still persist on this matter. There are several aspects that can be addressed in order to highlight the quality with which you can develop a training process supported by the information and communication technology, ICT.

One of the structural aspects in the formative process of engineers in this methodology, is the practice of laboratory; from this perspective a real practice stage appears, although remote, called Laboratory of Remote Access or Remote Laboratory $(R L)$. In this sense, the question that arises is the design of an architecture at engineering level for $R L$, which eliminates the barrier of space and time, reduces costs in infrastructure and has a major universality grade in the access, without sacrificing the quality that demands the rigorous exercise of the laboratory practice in the Engineer formation.

Keywords: architecture, competitiveness, distance education, engineering, globalization, ICT

\section{INTRODUCCIÓN}

El mundo ha cambiado, las dinámicas en que se mueve la sociedad y su entorno han sufrido importantes transformaciones, las suficientes para creer que es irreversible. En el actual contexto de globalización, las organizaciones y las personas están obligadas a responder a los retos que demandan el nuevo entorno, de lo contrario estarán condenadas al rezago. La educación, principalmente la educación superior por ser uno de los elementos dinamizadores y transformadores de la sociedad, es la primera en ser llamada a asumir los nuevos cambios. En una sociedad globalizada y dinámica, la Educación Abierta y a Distancia, EaD, soportada en las Tecnología de la información y la Comunicación, TIC, es uno de los componentes más efectivos para responder a la necesidad de buscar una sociedad incluyente, desarrollada y competitiva en el contexto global; no obstante, esta metodología exige responder con suficiente claridad y objetividad algunas dudas que puedan existir acerca de los procesos formativos que se desarrollan allí.

Uno de los aspectos críticos, objeto de cuestionamientos en la comunidad académica, es la formación de Ingenieros bajo la modalidad de la Educación Abierta y a Distancia, soportada en TIC, y específicamente, el desarrollo de la práctica de laboratorio. Desde esta óptica se ha planteado el diseño de una arquitectura, a nivel de Ingeniería, para Laboratorio de Acceso Remoto, que elimine las barreras de espacio y tiempo, reduzca costos en infraestructura y ofrezca una mayor posibilidad de acceso.

Para este proyecto se tuvo en cuenta la investigación descriptiva y correlacional. Por tanto, en un primer momento, se hizo una lectura de la percepción de la población objetivo para, de esta manera, medir el impacto que se puede generar. Seguidamente y con base en una lectura de recursos disponibles se construyó el diseño de la arquitectura y, a partir de este, uno de los elementos funcionales.

\section{Pertinencia de la EaD}

En la última década, la sociedad ha sufrido profundas transformaciones, que han cambiado la forma como el ser humano realiza sus actividades. Las Tecnologías de la Información y la Comunicación (TIC) han tenido un desarrollo explosivo en la última parte del siglo XX y el comienzo del siglo XXI, al punto de que han dado forma a lo que se denomina "Sociedad del Conocimiento" 0 "de la Información". Prácticamente no hay un solo ámbito de la vida humana que no se haya visto 
impactada por este desarrollo: la salud, las finanzas, los mercados laborales, las comunicaciones, el gobierno, la productividad industrial, etc. [1].

En medio de esta intensa dinámica, la educación y en especial la educación superior debe responder de una manera pertinente frente a este nuevo contexto, siendo la formación en Ingeniería una de las áreas llamadas a asumir este reto con calidad, responsabilidad y ética. En la ingeniería se sustenta la construcción de capacidad en infraestructura e innovación que da como resultado competitividad en un mercado cada vez más global [2]. Las Tecnologías de la Información y la Comunicación son quizás el elemento catalizador con mayor impacto en las dinámicas transformadoras; en este sentido, la educación superior, como componente transversal de los diferentes sectores, debe estar en la capacidad de incorporar las TIC, como parte estructural del soporte en su desarrollo. No obstante, sigue siendo un recurso que no se maneja de la manera más adecuada y pertinente; se utiliza principalmente a nivel de redes sociales, pues muchos no creen en las oportunidades que pueden brindar para el fortalecimiento de los procesos académicos, principalmente en la Educación Abierta y a Distancia.

Cuando se hace un análisis de la educación, se pueden considerar tres escenarios: uno, la educación es un derecho fundamental, que le permite al ser humano acceder a los otros derechos y con ellos mejorar las condiciones de vida. La Organización de las Naciones Unidas para la Educación, la Ciencia y la Cultura, Unesco, ha recibido el mandato de sus Estados Miembros para abordar los temas clave, las tensiones y las posibilidades al alcance de las políticas públicas que permitan aprovechar el potencial de las TIC a favor de la educación y el desarrollo [1]. Desde un segundo escenario, el mundo desarrollado e industrializado que gira alrededor de los negocios, se mueve dentro de unas dinámicas en las que el tiempo no es el mejor aliado, pero sí la necesidad de construir conocimiento, de innovar; es decir, de continuar en un proceso de formación, que les permita hacerse más competentes e idóneos para asumir permanentemente los retos. En este caso, el modelo de educación tradicional no propicia el mejor contexto para responder a estas necesidades, por un lado, por las limitaciones financieras de algunos gobiernos que no les permite contar con la infraestructura física necesaria para educar a la población y, por otro, el tiempo, que no les permite desplazarse a lugares físicos para recibir una clase. Un tercer escenario, y un poco más global, radica en que actualmente es importante comprender que la información está disponible; se puede decir que es pública, salvo algunas pocas excepciones. Por tanto, para construir conocimiento se deben manejar dinámicas diferentes; ya no es el escenario profesorestudiante, en el que es el profesor el que lo sabe todo; el rol del docente es de guía, acompañante o asesor y quien, a través de recursos digitales, deberá estar en condiciones de construir Ambientes Virtuales de Aprendizajes, AVAs, considerando una pedagogía y didáctica que se fundamentan en una selección o curaduría de contenidos, una estructuración lógica de actividades académicas que permitan desarrollar las competencias generales y específicas según los propósitos de formación del respectivo plan de formación.

Como se considera que aprender es gestionar el conocimiento o el desconocimiento, aquí se propone que los entornos deben tener esta característica. Además el centro es el estudiante, en la gestión de los conocimientos y la de sus aprendizajes. [3].

En este sentido, la Educación Abierta y a Distancia soportada en las Tecnologías de la Información y la Comunicación, TIC, es la respuesta más pertinente en el nuevo contexto de globalización. Sin embargo, la incorporación de las TIC por sí solas no garantizan la equidad y la inclusión, como tampoco la calidad en los procesos educativos; se requiere de un modelo pedagógico con una didáctica definida y unas estrategias de aprendizaje que 
permitan, desde su complejidad, desarrollarse en Ambientes Virtuales de Aprendizaje, bajo unos escenarios interactivos y dinámicos.

\section{Diseño de la arquitectura de Un Laboratorio Remoto (LR)}

En la formación de Ingenieros bajo la modalidad de Educación Abierta y a Distancia soportada en TIC, uno de los aspectos de importante consideración es el desarrollo del componente práctico. Gracias a los avances del proyecto Aplicativo de software remoto soportado en (IP) para el desarrollo de aplicaciones orientadas al control de nivel y temperatura,se ha comprendido que una de las formas más adecuadas, pero no la única, de clasificar el componente práctico, tiene que ver con la manipulación que se haga de las variables involucradas en la práctica [4]. En la Fig. 1 se puede apreciar, entonces, la clasificación de las prácticas de laboratorio.

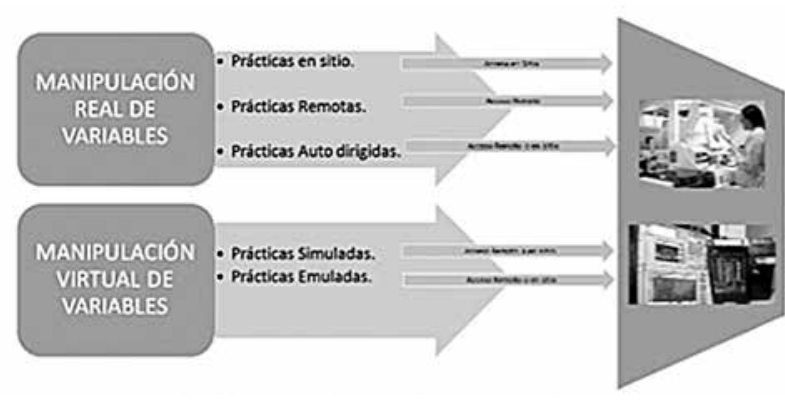

Fig. 1 Categorías y tipos de laboratorio

Siendo el Laboratorio Remoto (LR) una evolución del Laboratorio Virtual (LV), este busca responder a la necesidad de no alejar la práctica de laboratorio de la realidad, [5] como requerimiento fundamental en la formación del Ingeniero, pero sin la demanda de desplazamientos físicos que generan pérdida de tiempo para el usuario y costos significativos.
Lo anterior exige una infraestructura tecnológica, por lo que se hace necesario diseñar una arquitectura que defina unas unidades funcionales, redes de telecomunicaciones, protocolo de comunicación y desarrollo de un software que permita la integración del sistema y el ambiente de interacción usuario - LR., de manera transparente y en tiempo real.

Desde esta perspectiva, se encontró que un LR debe contar con una arquitectura compuesta por una capa de gestión interna que actúe como una especie de compilador e interprete la señalización desde el controlador del laboratorio a través de la tarjeta de interfaz inteligente que establece comunicación Full-Duplex con el servidor. Internamente, esta tarjeta cuenta con la tarjeta de adquisición de datos y, de igual forma, recibe la señalización desde el usuario (estudiante) ubicado en la red externa y la envía hasta el controlador del laboratorio [6]

La capa de acceso proporciona conectividad a los usuarios y hace uso del protocolo H.248 con RTCP y RTSP con canales dedicados pero sincronizados. La capa de distribución se utiliza para enviar el tráfico de una red local a otra, utilizando tecnología SDH o ATM, [7] entre diferentes laboratorios que pueden estar conectados. Por último, la capa de núcleo representa una capa troncal de alta velocidad entre las redes dispersas. El tráfico de los usuarios se inicia en la capa de acceso y pasa por las demás capas si se necesita utilizar la funcionalidad de las mismas.

Básicamente, los routers son computadoras especializadas, que requieren una CPU y una memoria para almacenar datos de forma temporal y permanente a fin de ejecutar las instrucciones del sistema operativo, como la inicialización del sistema y las funciones de routing y de switching . 


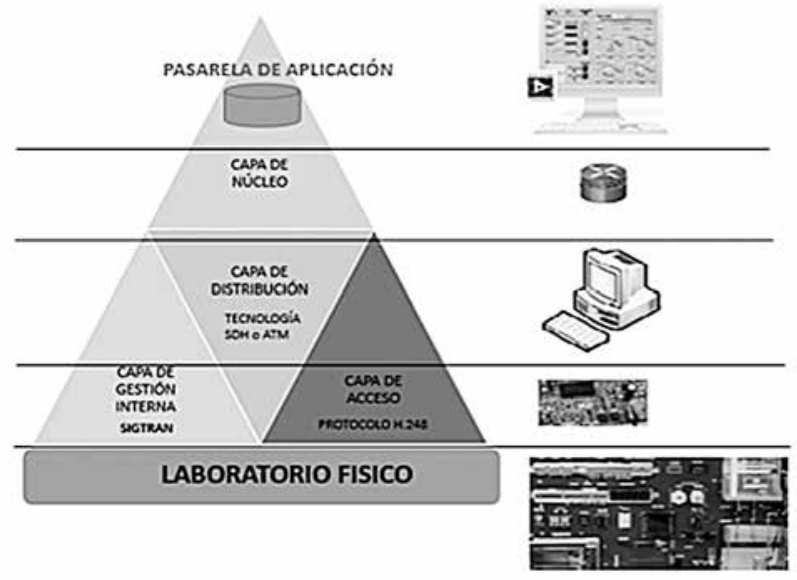

Fig. 2 Arquitectura de $L R$

La arquitectura del Laboratorio Remoto (LR) según figura 2 , debe contar con unas características técnicas mínimas que garanticen tolerancia a fallas, escalabilidad, calidad del servicio y seguridad, bajo una mirada prospectiva de ese Modelo de Laboratorio Remoto. Desde esta perspectiva, se pueden considerar las siguientes características: el flujo o transferencia de datos debe estar basado en paquetes; debe haber separación entre la provisión del servicio y el transporte y proveer interfaces abiertas, soportar una amplia gama de aplicaciones, servicios y mecanismos, tanto en tiempo real como en tiempo no real, y multimedios; debe permitir integrar redes precedentes (PSTN/ISDN y otras) a través de interfaces abiertas; permitir acceso de los usuarios a servicios ofrecidos por diferentes proveedores; debe trabajar con un mismo perfil de servicio para un usuario en toda la red; debe garantizar la convergencia de los servicios fijos y móviles y la independencia de las funciones de un servicio de las tecnologías de transporte subyacentes y debe soportar múltiples tecnologías de última milla.

Dado la evolución o desarrollo en las Tecnología de las Telecomunicaciones y los requerimientos que demanda la Arquitectura del Laboratorio Remoto (LR), este se desarrolla bajo los estándares y protocolos TCP/IP. Los protocolos TCP/
IP están incluidos en la capa de internet hasta la capa de aplicación, cuando se hace referencia al modelo TCP/IP. Los protocolos de capa inferior de la capa de enlace de datos o de la capa de acceso a la red son responsables de enviar el paquete IP a través del medio físico. Estos protocolos de capa inferior son desarrollados por organismos de estandarización, como el IEEE.

Los protocolos IP, HTTP y DHCP son todos parte de la de protocolos de internet, conocida como protocolo de control de transmisión/IP (TCP/IP). La de protocolos TCP/IP es un estándar abierto, lo que significa que estos protocolos están disponibles para el público, sin cargo, y cualquier proveedor puede implementarlos en su hardware o software. Para optimizar el ancho de banda en la red dedicada a la Arquitectura del LR, esta debe estar organizada para que el tráfico se mantenga en el nivel local y no se propague innecesariamente a otras partes de la red. El uso del modelo de diseño jerárquico de cuatro capas ayuda a hacer más eficiente el ancho de banda.

\section{Referencias}

[1] UNESCO, "Enfoque Estratégico Sobre Tics En Educación En América Latina y El Caribe" , 2013, p. 62.

[2] M. Duque and J. Celis, Educación en ingeniería para la ciudadanía, la innovación y la competitividad en Iberoamérica, 2012. pp.58-62.

[3] D. del Arco, "Tesis Doctoral Modelo para la Creación de Entornos de Aprendizaje basados en técnicas de Gestión del Conocimiento", 2003.

[4] R. Con, N. D. E. Carbono, and O. Mecanicamente, "Revista Colombiana de Materiales N.5 Edición Especial Artículos Cortos" pp. 93-99.

[5] C. Riman, "A Remote Robotic Laboratory Experiment Platform with Error Management", vol. 8, no. 1, 2011, pp. 242-248.

[6] R. Zamora, "Laboratorios Remotos: Actualidad y tendencias futuras", vol. № 51, no. 51, pp. 113-118, 2012.

[7] A. Atlánticos, C. O. N. Sotobosque, D. E. I. Y. A. Veces, and D. E. T. Quercion, "Universidad Politécnica de Madrid Escuela Técnica Superior de Ingenieros De Montes", 2011. 
